\title{
Validación de la Escala de Satisfacción con la Vida y su relación con las dimensiones del autoconcepto en universitarios peruanos
}

\author{
Amparo Oliver \\ Laura Galiana \\ Universitat de València, \\ Valencia, España \\ Varinia Bustos \\ Universidad ESAN, \\ Lima, Perú
}

Recibido: 21 de noviembre del 2017 / Aprobado: 25 de julio del 2018

doi: 10.26439/persona2018.n021.3018

La satisfacción con la vida ha sido objeto de innumerables investigaciones, la mayoría de las cuales la han evaluado con la Escala de Satisfacción con la Vida. Recientemente, se ha puesto interés en su relación con el autoconcepto, que parece depender del contexto cultural. Los objetivos de este estudio fueron conocer las propiedades de la Escala de Satisfacción con la Vida en una muestra de estudiantes peruanos, y estudiar la relación de la satisfacción vital con el autoconcepto en este mismo contexto. Se recogió una muestra de 527 estudiantes universitarios de Lima (Perú). En primer lugar, se estimó y evaluó un análisis factorial confirmatorio, y se llevaron a cabo estimaciones de fiabilidad de la escala y sus items. Posteriormente, se puso a prueba un modelo de ecuaciones estructurales MIMIC, en el que el autoconcepto predecía la satisfacción con la vida. Los resultados ofrecen evidencia de buenas propiedades psicométricas de la escala en su uso en estudiantes peruanos, y comprueban la relación de este constructo con las dimensiones académica, familiar y física del autoconcepto.

satisfacción con la vida / bienestar / modelo MIMIC / autoconcepto

Correo electrónico: oliver@uv.es 


\section{Validation of the Satisfaction with Life Scale and its relationship with the dimensions of self-concept in Peruvian university students}

Life satisfaction has been the object of several studies, mostly assessed with the Satisfaction with Life Scale (SWLS). Recently, interest has been shown in the relationship between life satisfaction and self-concept, because life satisfaction seems to depend on the cultural context. The purpose of this research was to study the properties of the SWLS in a sample of Peruvian students, and to analyze the relationship between life satisfaction and self-concept in the Peruvian context. The study population consisted of 527 university students from Lima, Peru. Firstly, a confirmatory factor analysis was estimated and assessed, and reliability estimates of the scale and its items were calculated. Subsequently, a MIMIC structural equation model in which self-concept predicted life satisfaction was tested. The results show appropriate psychometric properties of the scale in Peruvian students, and demonstrate the relationship of this construct with the academic, family and physical dimensions of self-concept. 


\section{INTRODUCCIÓN}

El bienestar, concebido como "el nivel de calidad de vida, por ejemplo, la medida en que el placer y la satisfacción caracterizan la existencia humana y la medida en que las personas podemos evitar el sufrimiento" (Andrews, 1974, p. 280), ha acumulado gran cantidad de investigación a lo largo de los años (Abbott, Ploubidis, Huppert, Kuh y Croudace, 2010; Burns y Machin, 2009; Dolan y White, 2007; Huppert, Keverne y Bayliss, 2005; Ryan y Deci, 2001; Seligman, 2002; Snyder y López, 2001).

Desde un primer momento, se han identificado dos partes diferentes del constructo de bienestar subjetivo: la parte afectiva, habitualmente dividida entre afecto positivo y negativo (Diener y Emmons, 1984), y el componente cognitivo, que se conoce como satisfacción con la vida (Andrews y Withey, 1976). Así pues, la satisfacción con la vida se refiere a un proceso cognitivo, de emisión de juicio, en el que la persona evalúa de manera global su calidad de vida, de acuerdo con unos criterios autoimpuestos (Diener, Emmons, Larsen y Griffin, 1985; Shin y Johnson, 1978). Se trata de un juicio cognitivo consciente, basado en la comparación de la vida de uno mismo frente a unos estándares escogidos por la persona, que conducen a una evaluación global de la vida (Pavot y Diener, 1993).

Dada la relevancia del constructo, durante las últimas décadas se han desarrollado numerosos instrumentos para su medida. Estos incluyen desde indicadores simples de satisfacción general con la vida hasta escalas breves, como el Índice de Bienestar y Satisfacción con la Vida
(Bigot, 1974), o escalas complejas y multidimensionales, como la Escala Moral del Centro Geriátrico de Filadelfia (Lawton, 1975). De todos ellos destaca la Escala de Satisfacción con la Vida (Diener et al., 1985), que evalúa, mediante cinco ítems, la satisfacción global con la vida, en un formato de respuesta de tipo Likert. Esta escala es, sin lugar a dudas, la más usada, pues se ha traducido a diversas lenguas $y$ aplicado en diversos tipos de poblaciones (Pavot y Diener, 2008). En el caso de la versión española, se encuentran investigaciones en España (Bendayan, Blanca, Fernández-Baena, Escobar y Trianes, 2013; Díaz-Morales, 2001; Grao-Cruces, Nuviala, Fernández-Martínez y Pérez-Turpin, 2014; Jiménez-Moral, Zagalaz, Molero, PulidoMartos y Ruiz, 2014; Singelis et al., 2006; Videra-García y Reigal-Garrido, 2013), Argentina (Castro y Díaz-Morales, 2002) y Perú (Díaz-Morales y Martínez, 2004; Martínez, 2002, 2004).

Sin embargo, las investigaciones realizadas con este instrumento no se han limitado al estudio de sus propiedades psicométricas, sino que han aportado evidencia sobre la relación de la satisfacción con la vida con los más diversos constructos, como, por ejemplo, extroversión, introversión, neuroticismo, autoestima, euforia, disforia, deseabilidad social, salud percibida, generatividad, control percibido o apoyo social (Arrindell, Heesink y Feij, 1999; Cabañero et al., 2004; Diener et al., 1985; Harrington y Loffredo, 2001; Infurna, Ram, Wagner, Gerstorf y Schupp, 2011; Lucas, Diener y Suh, 1996; McAdams, Aubin y Logan, 1993; Pavot y Diener, 1993; Theurer y Wister, 2010). 
De especial relevancia nos resulta su relación con la autoestima, que ha recobrado interés en los últimos años, gracias al surgimiento del nuevo paradigma de la psicología positiva (Seligman y Csikszentmihalyi, 2000). Desde este acercamiento al estudio de las experiencias y los rasgos positivos (Duckworth, Steen y Seligman, 2005), el autoconcepto se ha mostrado clave para el bienestar y la satisfacción con la vida del ser humano (Diener y Diener, 2009; Leung y Leung, 1992; Rey, Extremera y Pena, 2011). Sin embargo, a pesar de que en la literatura se ha recogido una amplia evidencia sobre la relación entre el autoconcepto y la satisfacción con la vida (Campbell, 1981; Chen, Lee y Stevenson, 2006; Diener et al., 1985; Joshanloo y Afshari 2011; Lucas et al., 1996; Pavot y Diener, 1993), la mayor parte de esta corresponde al ámbito anglosajón (Suh, 2000). De hecho, algunos estudios indican que la fuerza de la relación entre autoconcepto y bienestar varía según el marco cultural (Suh, 2000), lo que no es de extrañar si tenemos en cuenta que el propio autoconcepto también varía transculturalmente (Markus y Kitayama, 1991). Ejemplos claros son los estudios de Brown y Kobayashi (2002, 2003) o de Miller, Wang, Sandel y Cho (2002), en Japón y Taiwán, respectivamente, donde los niveles elevados de autoconcepto se entenderían como una baja capacidad de autocrítica. Otro estudio más actual ha comprobado cómo los adolescentes chinos informan de una satisfacción con la vida mucho menor que los estadounidenses. También hay autores que explican la tendencia de los asiáticos a evitar respuestas extremas superiores (Cummins, 2013). De esta forma, en culturas colectivistas, el autoconcepto podría ser poco importante o, incluso, poco deseable (para más detalle, véase Diener y Diener, 2009).

Dado lo expuesto hasta ahora, el presente trabajo tiene un doble objetivo: de un lado, estudiar las propiedades psicométricas de la Escala de Satisfacción con la Vida en una muestra de estudiantes universitarios peruanos, contexto en el que hasta el momento no se cuenta con este tipo de estudios; $y$, de otra parte, conocer cómo se relaciona este constructo con la autoestima en el Perú, un país en el que actualmente confluyen la cultura occidental, con sus características individualistas, y las tradiciones de tipo más colectivistas, basadas en la importancia del núcleo familiar y la red social.

\section{Método}

\section{Participantes}

La muestra estuvo compuesta por 527 estudiantes de dos universidades de Lima (Perú). De ellos, el 57,9 \% fueron mujeres. El 29,6 \% tenía entre 19 y 20 años, el 38,0\% entre 21 y 22 , el $20,7 \%$ entre 23 y 24 , el $8,2 \%$ entre 25 y 30 , y tan solo un 3,5\% tenía más de 30 años. Finalmente, en el momento del presente trabajo, el $28,7 \%$ estudiaba Contabilidad; el 18,4\%, Administración; el 15,6\%, Administración y Marketing; el 13,5 \%, Economía; el 7,8 \%, Psicología Organizacional; el 6,8\%, Psicología del Consumidor; el 4,8 \%, Administración y Finanzas; el 2,5\%, Derecho Comercial; el $1,1 \%$, Ingeniería Industrial; y el 0,8 \% restante, otras carreras. 


\section{Instrumentos}

Además de las preguntas sobre los datos sociodemográficos expuestos, la encuesta también incluyó, entre otros, los siguientes instrumentos de evaluación:

a. Escala de Satisfacción con la Vida (Diener et al., 1985). Desarrollada por Diener y sus colaboradores (1985), es uno de los instrumentos más utilizados en este campo de la investigación (Pavot y Diener, 2008). Está formado por cinco ítems que evalúan un único constructo: el sentimiento general de satisfacción de la persona con su vida como un todo (Diener et al., 1985). Estos ítems tienen escala de tipo Likert de cinco anclajes: desde 1 (totalmente en desacuerdo) hasta 5 (totalmente de acuerdo). La consistencia interna, así como el resto de propiedades psicométricas de este instrumento para esta muestra, se detallan en la sección de resultados.

b. Escala Autoconcepto Forma 5 (García y Musitu, 1999). Este instrumento evalúa cinco dimensiones del autoconcepto: académica, social, emocional, familiar y física, cada una con seis ítems. Estos puntúan en una escala de tipo Likert, que admite 11 posibilidades de respuesta $(0=$ nunca y $10=$ siempre $)$. El alfa de Cronbach fue de .81 para la dimensión de autoconcepto académico, .73 para el autoconcepto social, .82 para el emocional, .76 para el familiar y .75 para el físico.

\section{Procedimiento}

El presente trabajo sigue un diseño de encuesta transversal. Se llevaron a cabo encuestas de lápiz y papel en dos universidades públicas en el Perú. En ambos casos, se contó con la aprobación de los órganos de gestión correspondientes. La participación de los estudiantes fue totalmente voluntaria e informada.

El pase se realizó en horario de clases, en las propias aulas de los respectivos centros. En todo momento, se contó con la presencia de entrevistadores formados en las aulas. Los estudiantes tardaron una media de 30 minutos en completar la encuesta.

En cuanto a los análisis de datos, en primer lugar, y con el objetivo de estudiar la fiabilidad de las medidas, se calcularon estadísticos descriptivos para los ítems de las diferentes escalas (medias y desviaciones típicas) y el alfa de Cronbach. El alfa es el índice de fiabilidad más ampliamente utilizado y conocido por la comunidad científica para evaluar la fiabilidad de tests y escalas, con valores de .70 a .79 , considerados moderados, y de .80 o superiores, interpretados como alta fiabilidad (Nunnally y Bernstein, 1994; Cicchetti, 1994; Clark y Watson, 1995). Además, se incluyó la homogeneidad de los ítems.

En segundo lugar, se procedió al estudio de la validez de constructo de la medida. Para ello, se especificó, estimó y evaluó un modelo de ecuaciones estructurales, en concreto, un análisis factorial confirmatorio (AFC), en el que se probó la estructura original propuesta por los autores (Diener et al., 1985).

Finalmente, para evaluar la validez criterial de la escala, así como la relación entre la satisfacción con la vida y el autoconcepto, se especificó un modelo de ecuaciones 
estructurales MIMIC (Multiple Indicators, Multiple Causes), en el que las cinco dimensiones del autoconcepto predecían la satisfacción con la vida. Este tipo de modelos permite pronosticar la satisfacción con la vida en un contexto libre de error de medida en la variable dependiente y teniendo en cuenta relaciones entre los predictores e indicadores (ítems) particulares de la satisfacción con la vida, si las hubiese.

La adecuación de los modelos se evaluó mediante diversos criterios ( $\mathrm{Hu}$ y Bentler, 1995; Tanaka, 1993). En primer lugar, se calculó el estadístico chi-cuadrado $\left(c^{2}\right)$, el índice de ajuste original de los modelos de ecuaciones estructurales (Wang y Wang, 2012). Este estadístico evalúa la magnitud de la discrepancia entre las matrices de varianzas y de covarianzas de la muestra y las del modelo estimado, aunque sufre de diversas limitaciones: (1) es sensible al tamaño muestral; (2) es probable que las muestras pequeñas no sigan una distribución chi-cuadrada; (3) es sensible al tamaño de las correlaciones; y (4) es un estadístico muy sensible a la asunción de normalidad multivariada.

Para hacer frente a estas limitaciones, se han desarrollado diversos índices que evalúan la bondad del ajuste de los modelos de ecuaciones estructurales. Uno de ellos es el índice de ajuste comparativo (Comparative Fit Index, CFI), desarrollado por Bentler (1990), que compara el modelo especificado con el modelo nulo, que asume covarianzas iguales a cero entre las variables observadas. El CFI puede tener valores entre 0 y 1.00, que corresponden, respectivamente, al peor y al mejor ajuste posible; los valores superiores a .90 , e, idealmente, los mayores a .95 , indican un buen ajuste (Hu y Bentler, 1998, 1999). Otro índice, también muy utilizado, es la raíz cuadrática del error de aproximación (Root Mean Square Error of Approximation, RMSEA). El RMSEA evalúa la falta de ajuste del modelo especificado a la población. $\mathrm{Hu}$ y Bentler (1998) sugieren que valores de RMSEA iguales o inferiores a .08 son indicadores de un buen ajuste del modelo.

\section{Resultados}

La consistencia interna fue adecuada, con un alfa de Cronbach de .78. La información sobre la consistencia interna de los ítems puede observarse en la tabla 1. 
Tabla 1

Estadísticos descriptivos y homogeneidad de los ítems de la Escala de Satisfacción con la Vida

\begin{tabular}{llll}
\hline Ítem & $\mathrm{M}$ & $\mathrm{DT}$ & $\mathrm{a}_{n-i}$ \\
\hline $\mathrm{I} 1$ & 3,55 & 0,97 & .73 \\
$\mathrm{I} 2$ & 3,73 & 0,89 & .74 \\
$\mathrm{I} 3$ & 3,75 & 1,00 & .69 \\
$\mathrm{I} 4$ & 2,95 & 1,32 & .77 \\
$\mathrm{I} 5$ & 3,70 & 0,93 & .73 \\
\hline
\end{tabular}

Nota: $\mathrm{M}=$ media; $\mathrm{DT}=$ desviación típica; $\alpha_{n-i}=$ alfa si se elimina el elemento.

En cuanto a la estructura factorial, el análisis factorial confirmatorio realizado sobre la Escala de Satisfacción con la Vida ofreció unos índices de ajuste adecuados $\left(c^{2}(5)=19.464, \mathrm{p}=.001, \mathrm{CFI}\right.$ $=.985$, RMSEA $=.075$ [.042-.111]). En cuanto al ajuste analítico, este también fue apropiado, con saturaciones factoriales elevadas y estadísticamente significativas, que oscilaron entre .61 (ítem 4, "Si pudiera vivir mi vida otra vez, la repetiría tal y como ha sido") y .84 (item 3, "Estoy satisfecho con mi vida"), como se puede observar en la figura 1 .

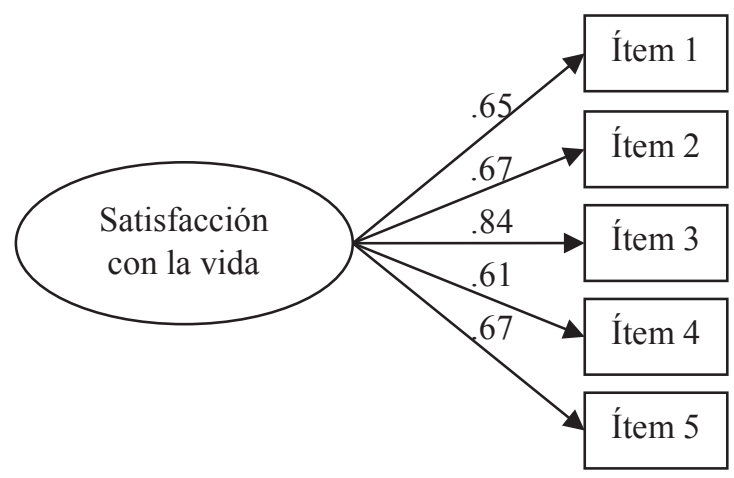

Nota: Todas las cargas factoriales fueron estadísticamente significativas $(p<.05)$.

Figura 1. Saturaciones factoriales del modelo de ecuaciones estructurales propuesto para la Escala de Satisfacción con la Vida 
Finalmente, por lo que respecta a las relaciones entre la satisfacción con la vida y las dimensiones del autoconcepto, el ajuste del modelo MIMIC fue adecuado $\left(c^{2}(5)\right.$ $=62,245, \mathrm{p}<.001, \mathrm{CFI}=.964$, RMSEA $=.054$ [.037-.071]). Muestra relaciones positivas y estadísticamente significativas entre la satisfacción con la vida y el autoconcepto académico, familiar y físico, como se puede observar en la figura 2. La capacidad explicativa del modelo sobre la satisfacción con la vida fue del 23,8 \%.

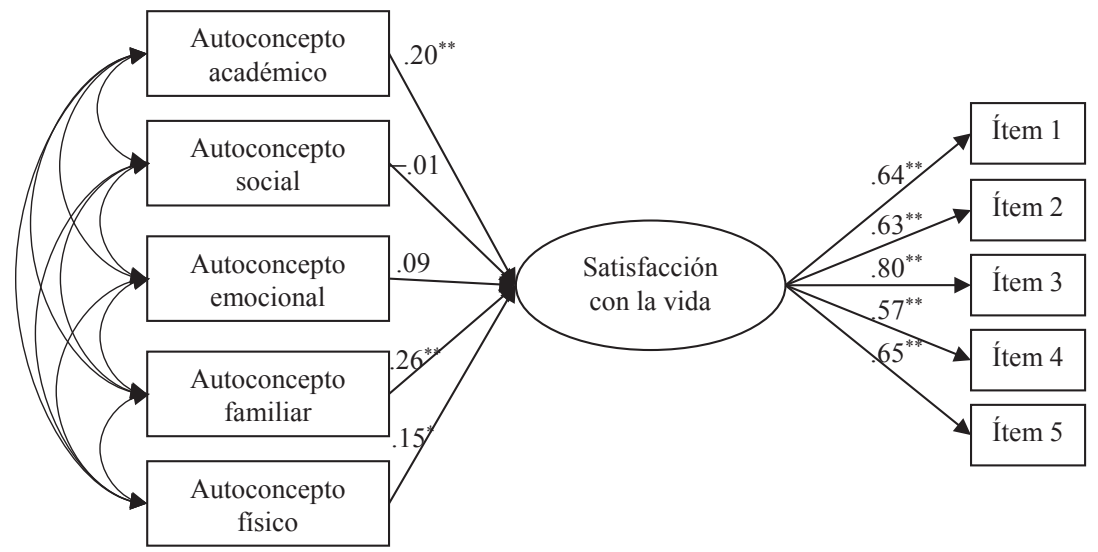

Notas: " $p<.05 ;{ }^{* *} p<.01$. Los valores de las correlaciones entre las dimensiones del autoconcepto pueden consultarse en la tabla 2.

Figura 2. Modelo MIMIC

Tabla 2

Correlaciones entre las dimensiones del autoconcepto

\begin{tabular}{llllll}
\hline & A. académico & A. social & A. emocional & A. familiar & A. físico \\
\hline A. académico & - & & & & \\
A. social & $.37^{* *}$ & - & & & \\
A. emocional & $.18^{* *}$ & $.31^{* *}$ & - & & \\
A. familiar & $.28^{* *}$ & $.39^{* *}$ & $.26^{* *}$ & - & \\
A. físico & $.56^{* *}$ & $.35^{* *}$ & $.17^{* *}$ & $.32^{* *}$ & - \\
\hline
\end{tabular}

Notas: ${ }^{* *} p<.01$. 


\section{DISCUSIÓN Y CONCLUSIONES}

Como afirman diversas instituciones y psicómetras de reconocido prestigio, la validezno es una propiedad de los instrumentos en sí, sino de las puntuaciones en estos y de su interpretación. De esta forma, dicha propiedad debería establecerse o evaluarse para cada aplicación de un determinado instrumento de medida en cada contexto específico, pues diferentes contextos pueden comportar diferentes interpretaciones de las puntuaciones (American Educational Research Association, American Psychological Association, National Council on Measurement in Education, 2004; Messick, 1989). Esta lógica es la que ha guiado el primer objetivo del presente trabajo: conocer las propiedades psicométricas de la Escala de Satisfacción con la Vida (Diener et al., 1985) en una muestra de estudiantes universitarios peruanos, población en la que, hasta donde conocemos, no existen estudios previos de este tipo.

Para llevar a cabo esta meta, se han realizado distintos análisis estadísticos. En primer lugar, se estudió la consistencia interna de la escala, con resultados adecuados tanto para la escala en general como para los ítems en particular. De una parte, ninguno de los ítems perjudicó la fiabilidad de la escala, pues su eliminación produjo en todos los casos un decremento del alfa. De otra parte, el valor del alfa de Cronbach fue alto (Nunnally y Bernstein, 1994; Cicchetti, 1994; Clark y Watson, 1995) y conforme a los valores encontrados en estudios previos; por ejemplo, Singelis et al. (2006) hallaron valores que oscilaban entre .74 y .75 , mientras que Bendayan et al. (2013) informaron de un alfa de .71 .
En segundo lugar, se hipotetizó y estimó un análisis factorial confirmatorio para evaluar la validez de constructo de la escala. Tanto el ajuste general del modelo como el ajuste analítico fueron adecuados, aportando evidencia de la estructura unidimensional de la escala, recogida previamente tanto por los autores originales (Diener et al., 1985; Pavot y Diener, 1993) como en sucesivas versiones en distintos idiomas (por ejemplo, Arrindell et al., 1999).

Finalmente, el modelo MIMIC aportó evidencia de la validez criterial de la escala, relacionando la satisfacción con la vida de los estudiantes universitarios peruanos y su autoconcepto. En concreto, este modelo mostró relaciones estadísticamente significativas y positivas entre la satisfacción con la vida y las tres dimensiones del autoconcepto: el autoconcepto familiar, el autoconcepto académico y el autoconcepto físico. Las dimensiones social y emocional, sin embargo, no predijeron de forma estadísticamente significativa la satisfacción con la vida.

Estos resultados son similares a los de estudios previos. Claro ejemplo es el estudio de Leung y Leung (1992), llevado a cabo en adolescentes chinos, en el que la satisfacción con la vida mostró relaciones más elevadas con las dimensiones de habilidad social (.21), habilidad académica (.20) y apariencia física (.18). Datos actuales avalan lo encontrado por estos autores, al señalar las relaciones entre la satisfacción con la vida y el autoconcepto académico (.30) (Chui y Wong, 2015).

En cuanto al autoconcepto físico, otros estudios han apuntado cómo el hecho de 
contar con un autoconcepto físico positivo resulta esencial para el desarrollo personal y social adecuado (Crocker et al., 2003; Crocker, Sabiston, Kowalski, McDonough y Kowalski, 2006; Delfabbro, Winefield, Anderson, Hammarström y Winefield, 2011; Grao-Cruces et al., 2014). VideraGarcía y Reigal Garrido (2013), por ejemplo, han señalado una relación directa entre el autoconcepto físico y la satisfacción con la vida en una muestra de 1648 adolescentes españoles. Rodríguez (2005), también en españoles y con mayor rango de edad (12-23 años), encontró una relación entre el bienestar psicológico y el autoconcepto físico. En contextos anglosajones existe, asimismo, abundante evidencia sobre esta relación, como, por ejemplo, la que muestra Delfabbro et al. (2001) en estudiantes australianos.

Por lo que respecta al apoyo social, este se ha considerado uno de los componentes más críticos para la determinación del bienestar de los estudiantes (Cho y Yu, 2015; Lee, Koeske, y Sales, 2004; Misra, Crist y Burant, 2003). Estudios recientes sostienen que aquellos que reciben mayor apoyo social de amigos y familia informan de mayor satisfacción con la vida (Kong y You, 2013; Kong, Zhao y You, 2012a, 2012b). En este contexto, los resultados del presente trabajo apuntarían al mayor peso de la familia frente al autoconcepto social más general en los estudiantes peruanos. Esta misma línea ya la indicaba el trabajo de Leung y Leung (1992). En este estudio, a pesar de que no se evaluó el autoconcepto familiar, la mayor relación se estableció entre la satisfacción con la vida y la relación con los padres (.49). De hecho, y ya en contexto de regresión múltiple, tan solo este predictor explicaba el $23,8 \%$ de la varianza en la satisfacción con la vida, frente a las demás dimensiones estadísticamente significativas, que añaden entre todas ellas un 2,2 \% de varianza explicada (Leung y Leung, 1992).

Por último, los estudios que relacionan el autoconcepto emocional y la satisfacción con la vida son más escasos y, en su mayoría, se han centrado en el papel de la autoeficacia emocional y el bienestar de los estudiantes (Bandura, Caprara, Barbaranelli, Gerbino y Pastorelli, 2003). En esta línea de investigación, parece clara la relación positiva entre la satisfacción con la vida y la autoeficacia emocional (véase, por ejemplo, Choi, Kluemper y Sauley, 2013).

Otra variable usualmente estudiada es el estrés emocional, que se ha relacionado negativamente con la satisfacción con la vida (Reile y Leinsalu, 2013). No se trata, sin embargo, de variables miméticas del autoconcepto emocional, pues en el primer caso (autoeficacia emocional) se evalúa la capacidad de la persona para aminorar los estados negativos y experimentar emociones positivas (Bandura et al., 2003), mientras que en el segundo (estrés emocional) se mide el estrés experimentado por la persona. Futuras investigaciones, pues, deberán retomar el estudio del autoconcepto emocional y su relación con la satisfacción con la vida.

Visto lo hasta aquí expuesto, y por lo que respecta al primer objetivo, podemos concluir que en el contexto de los estudiantes universitarios peruanos la satisfacción con la vida puede evaluarse con garantías mediante la Escala de Satisfacción con la Vida (Diener et al., 1985). En cuanto a la 
relación de este constructo con el autoconcepto y sus diversas dimensiones, parece que tanto factores más relacionados con las culturas colectivistas (como la familia) como otros más presentes en las culturas individualistas (como el físico) tienen reservado un papel importante. Futuros estudios son necesarios para ahondar en esta relación, con especial atención en el autoconcepto emocional.

\section{Referencias}

Abbott, R. A., Ploubidis, G. B., Huppert, H. A., Kuh, D., y Croudace, T. J. (2010). An Evaluation of the Precision of Measurement of Ryff's Psychological Well-Being Scales in a Population Sample. Social Indicators Research, 97, 357-373.

American Educational Research Association; American Psychological Association; National Council on Measurement in Education. (2004). Standards for Educational and Psychological Testing (2. ${ }^{\mathrm{a}}$ ed.). Washington, D. C.: American Educational Research Association.

Andrews, F. M. (1974). Social Indicators of Perceived Life Quality. Social Indicators Research, 1, 279-299.

Andrews, F. M., y Withey, S. B. (1976). Social Indicators of Well-Being: America's Perception of Life Quality. Nueva York, NY: Plenum.

Arrindell, W. A., Heesink, J., y Feij, J. A. (1999). The Satisfaction with Life Scale (SWLS). Appraisal with 1700 Health Young Adults in the Netherlands. Personality and Individual Differences, 26, 815-826.

Atienza, F. L., Pons, D., Balaguer, I., y García-Merita, M. L. (2000). Propiedades psicométricas de la Escala de Satisfacción con la Vida en adolescentes. Psicothema, 12, 331-336.

Bandura, A., Caprara, G.V., Barbaranelli, C., Gerbino, M., y Pastorelli, C. (2003). Role of Affective Self-Regulatory Efficacy on Diverse Spheres of Psychosocial Functioning. Child Development, 74, 769-782.

Bendayán, R., Blanca, M. J., Fernández-Baena, J. F., Escobar, M., y Trianes, M. V. (2013). New Empirical Evidence on the Validity of the Satisfaction with Life Scale in Early Adolescents. European Journal of Psychological Assessment, 29, 36-43.

Bentler, P. M. (1990). Comparative Fit Indexes in Structural Models. Psychological Bulletin, 107, 238-246.

Bigot, A. (1974). The Relevance of American Life Satisfaction Indices for Research on British Subjects Before and After Retirement. Age and Ageing, 2, 113-121.

Blais, M. R., Vallerand, R. J., Pelletier, L. G., y Briere, N. M. (1989). L'echelle de satisfaction de vie: validation canadienne-française du "Satisfaction with Life Scale". Canadian Journal of Behavioral Science, 21, 210-223. 
Brown, J. D., y Kobayashi, C. (2002). Self-Enhancement in Japan and America. Asian Journal of Social Psychology, 5, 145-168.

Brown, J. D., y Kobayashi, C. (2003). Introduction: Culture and the Self-Enhancement Bias. Journal of Cross-Cultural Psychology, 5, 492-495.

Burns, R. A., y Machin, M. A. (2009). Investigating the Structural Validity of Ryff's Psychological Well-Being Scales Across Two Samples. Social Indicators Research, 93, 359-375.

Cabañero, M. J., Richart, M., Cabrero, J., Orts, M. I., Reig, A., y Tosal, B. (2004). Fiabilidad y validez de la escala de satisfacción vital de Diener en una muestra de mujeres embarazadas y puérperas. Psicothema, 16, 448-455.

Campbell, A. (1981). The Sense of Well-Being in America: Recent Patterns and Trends. Nueva York, NY: McGraw-Hill.

Castro, A., y Díaz-Morales, J. F. (2002). Objetivos de vida y satisfacción vital en adolescentes españoles y argentinos. Psicothema, 4(1), 112-117.

Chen, C., Lee, S., y Stevenson, H. W. (1995). Response Style and Cross-Cultural Comparisons of Rating Scales among East Asian and North American Students. Psychological Science, 6, 170-175.

Cho, J., y Yu, H. (2015). Roles of University Support for International Students in the United States: Analysis of a Systematic Model of University Identification, University Support, and Psychological Well-Being. Journal of Studies in International Education, 19, 11-27.

Choi, S., Kluemper, D. H., y Sauley, K. S. (2013). Assessing Emotional Self-Efficacy: Evaluating Validity and Dimensionality with Cross-Cultural Samples. Applied Psychology: An International Review, 62, 97-123.

Chui, W. H., y Wong, M. Y. H. (2015). Gender Differences in Happiness and Life Satisfaction among Adolescents in Hong Kong: Relationships and Self-Concept. Social Indicators Research. doi: 10.1007/s11205-015-0867-z

Cicchetti, D. V. (1994). Guidelines, Criteria, and Rules of Thumb for Evaluating Normed and Standardized Assessment Instruments in Psychology. Psychological Assessment, 6, 284-290.

Clark, L. A., y Watson, D. (1995). Constructing Validity: Basic Issues in Objective Scale Development. Psychological Assessment, 7, 309-319.

Crocker, P. R. E., Sabiston, C. M., Forrestor, S., Kowalski, N., Kowalski, K. C., y McDonough, M. (2003). Predicting Change in Physical Activity, Dietary Restraint, and Physique Anxiety in Adolescent Girls: Examining Covariance in Physical Self-Perceptions. Canadian Journal of Public Health, 94(5), 332-337. 
Crocker, P. R. E., Sabiston, C. M., Kowalski, K. C., McDonough, M. H., y Kowalski, N. (2006). Longitudinal Assessment of the Relationship between Physical Self-Concept and Health-Related Behavior and Emotion in Adolescent Girls. Journal of Applied Sport Psychology, 18(3), 185-200.

Cummins, R. A. (2013). Measuring Happiness and Subjective Well-Being. En S. David, I. Boniwell y C. C. Ayers (Eds.), Oxford Handbook of Happiness (pp. 185-200). Oxford: Oxford University Press.

Delfabbro, P. H., Winefield, A. H., Anderson, S., Hammarström, A., y Winefield, H. (2011). Body Image and Psychological Well-Being in Adolescents: The Relationship between Gender and School Type. The Journal of Genetic Psychology, 172(1), 67-83.

Díaz-Morales, J. F. (2001). Diferencias intergrupales en el sistema de metas personales, satisfacción y rendimiento en adolescentes (tesis doctoral). Universidad Complutense de Madrid, España.

Díaz-Morales, J. F., y Martínez, U. (2004). Metas y satisfacción vital en adolescentes españoles y peruanos según sexo y nivel socioeconómico. Revista de Psicología de la PUCP, 22(1), 121-149.

Diener, E., y Diener, M. (2009). Cross-Cultural Correlates of Life Satisfaction and Self-Esteem. En E. Diener (Ed.), Culture and Well-being (pp. 71-91). Ámsterdam: Springer.

Diener, E., y Emmons, R. A. (1984). The Independence of Positive and Negative Affect. Journal of Personality and Social Psychology, 47, 1105-1117.

Diener, E., Emmons, R. A., Larsen, R. J., y Griffin, S. (1985). The Satisfaction with Life Scale. Journal of Personality Assessment, 49, 71-75.

Dolan, P., y White, M. (2007). How Can Measures of Subjective Well-Being Be Used to Inform Public Policy? Perspectives on Psychological Science, 2(1), 71-85.

Duckworth, A. L., Steen, T. A., y Seligman, M. E. P. (2005). Positive Psychology in Clinical Practice. Annual Review of Clinical Psychology, 1, 629-651.

García, J. F., y Musitu, G. (1999). AF5: Autoconcepto Forma 5. Madrid: TEA Ediciones.

Grao-Cruces, A., Nuviala, A., Fernández-Martínez, A., y Pérez-Turpin, J. A. (2014). Association of Physical Self-Concept with Physical Activity, Life Satisfaction and Mediterranean Diet in Adolescents. Kinesiology, 1, 3-11.

Harrington, R., y Loffredo, D. A. (2001). The Relationships between Life Satisfaction, Self-Consciousness, and the Myers-Briggs Type Inventory Dimensions. Journal of Psychology, 135, 439-450.

Hu, L. T., y Bentler, P. M. (1995). Evaluating Model Fit in SEM: Concepts, Issues, and Applications. En R. H. Hoyle (Ed.), Structural Equation Modeling. Concepts, Issues, and Applications (pp. 76-99). Londres: Sage. 
Hu, L. T., y Bentler, P. M. (1998). Fit Indices in Covariance Structure Modeling: Sensitivity to Under-Parameterized Model Misspecification. Psychological Methods, 3, 424-453.

Hu, L. T., y Bentler, P. M. (1999). Cutoff Criteria for Fit Indexes in Covariance Structure Analysis: Conventional Criteria versus New Alternatives. Structural Equation Modeling, 6, 1-55.

Huppert, F. A., Keverne, B., y Bayliss, N. (Eds.). (2005). The Science of Well-Being. Oxford: Oxford University Press.

Infurna, F. J., Ram, N., Wagner, G. G., Gerstorf, D., y Schupp, J. (2011). Long-Term Antecedents and Outcomes of Perceived Control. Psychology and Aging, 26, 559-575.

Jiménez-Moral, J. A., Zagalaz, M. L., Molero, D., Pulido-Martos, M., y Ruiz, J. R. (2013). Capacidad aeróbica, felicidad y satisfacción con la vida en adolescentes españoles. Revista de Psicología del Deporte, 22, 429-436.

Joshanloo, M., y Afshari, S. (2011). Big-five Personality Traits and Self-Esteem as Predictors of Life Satisfaction in Iranian Muslim University Students. Journal of Happiness Studies, 12, 105-113.

Lee, J. S., Koeske, G. F., y Sales, E. (2004). Social Support Buffering of Acculturative Stress: A Study of Mental Health Symptoms among Korean International Students. International Journal of Intercultural Relations, 28, 399-414.

Kong, F., y You, X. (2013). Loneliness and Self-Esteem as Mediators between Social Support and Life Satisfaction in Late Adolescence. Social Indicators Research, 110, 271-279.

Kong, F., Zhao, J., y You, X. (2012a). Emotional Intelligence and Life Satisfaction in Chinese University Students: The mediating Role of Self-Esteem and Social Support. Personality and Individual Differences, 53, 1039-1043.

Kong, F., Zhao, J., y You, X. (2012b). Social Support Mediates the Influence of Emotional Intelligence on Mental Distress and Life Satisfaction in Chinese Young Adults. Personality and Individual Differences, 53, 513-517.

Kwan, S. V. Y., Bond, M. H., y Singelis, T. M. (1997). Pancultural Explanations for Life Satisfaction: Adding Relational Harmony for Self-Esteem. Journal of Personality and Social Psychology, 73, 1038-1051.

Lawton, M. P. (1975). The Philadelphia Center Morale Scale: A Revision. Journal of Gerontology, 30, 85-89.

Leung, J., y Leung, K. (1992). Life Satisfaction, Self-Concept, and Relationship with Parents in Adolescence. Journal of Youth and Adolescence, 21, 653-665.

Lewis, C. A., Shevlin, M. E., Smekal, V., y Dorahy, M. J. (1999). Factor Structure and Reliability of a Czech Translation of the Satisfaction with Life Scale among Czech University Students. Studia Psychologica, 41, 239-244. 
Lucas, R. E., Diener, E., y Suh, E. (1996). Discriminant Validity of Well-Being Measures. Journal of Personality Assessment, 71, 3616-3628.

Markus, H. R., y Kitayama, S. (1991). Culture and the Self: Implications for Cognition, Emotion, and Motivation. Psychological Review, 98, 224-253.

Martínez, P. (2002). Perspectiva temporal futura en adolescentes de Lima, Perú (tesis de maestría). Universidad Autónoma de Barcelona, España.

Martínez, P. (2004). Perspectiva temporal futura y satisfacción con la vida a lo largo del ciclo vital (tesis doctoral). Universidad Autónoma de Barcelona, España.

McAdams, D. P., Aubin, E. S., y Logan, L. (1993). Generativity among Young, Midlife, and Older Adults. Psychology and Aging, 8, 221-230.

Messick, S. (1989). Validity. En R. L. Linn (Ed.), Educational Measurement (pp. 13-103). Nueva York, NY: Macmillan.

Miller, P. J., Wang, S., Sandel, T., y Cho, G. E. (2002). Self-Esteem as Folk Theory: A Comparison of European American and Taiwanese Mothers' Beliefs. Parenting: Science and Practice, 2, 209-239.

Misra, R., Crist, M., y Burant, C. J. (2003). Relationships among Life Stress, Social Support, Academic Stressors, and Reactions to Stressors of International Students in the United States. International Journal of Stress Management, 10, 137-157.

Nunnally, J., y Bernstein, I. H. (1994). Psychometric Theory (3. a ed.). Nueva York, NY: McGraw-Hill.

Pavot, W., y Diener, E. (1993). Review of the Satisfaction with Life Scale. Psychological Assessment, 5, 164-172.

Pavot, W., y Diener, E. (2008). The Satisfaction with Life Scale and the Emerging Construct of Life Satisfaction. The Journal of Positive Psychology, 3(2), 137-152.

Reile, R., y Leinsalu, M. (2013). Differentiating Positive and Negative Self-Rated Health: Results from a Cross-Sectional Study in Estonia. International Journal of Public Health, 58, 555-564.

Rey, L., Extremera, N., y Pena, M. (2011). Perceived Emotional Intelligence, Self-Esteem and Life Satisfaction in Adolescents. Psychosocial Intervention, 20, 227-234.

Rodríguez, A. (2005). Felicidad subjetiva y dimensiones del autoconcepto físico. Revista de Psicodidáctica, 10(1), 131-138.

Ryan, R., y Deci, E. L. (2001). On Happiness and Human Potentials. A Review of Research on Hedonic and Eudaimonic Well-Being. Annual Review of Psychology, 52, 141-166.

Seligman, M. E. P. (2002). Authentic Happiness. Londres: Nicholas Brealey. 
Seligman, M. E. P., y Csikszentmihalyi, M. (2000). Positive Psychology: An Introduction. American Psychologist, 55(1), 5-14.

Shin, D. C., y Johnson, D. M. (1978). A Vowed Happiness as an Overall Assessment of the Quality of Life. Social Indicators Research, 5, 475-492.

Singelis, T. M., Yamada, A. M., Barrio, C., Laney, J. H., Her, P., Ruiz-Anaya, A., y Terwillinger, S. (2006). Metric Equivalence of the Bidimensional Acculturation Scale, the Satisfaction with Life Scale, and the Self-Construal Scale Across Spanish and English Language Versions. Hispanic Journal of Behavioral Sciences, 28, 231-244.

Snyder, C. R., y López, S. J. (Eds.). (2001). Handbook of Positive Psychology. Nueva York, NY: Oxford University Press.

Suh, E. M. (2000). Self, the Hyphen between Culture and Subjective Well-Being. En E. Diener y E. M. Suh (Eds.), Culture and Subjective Well-Being (pp. 63-86). Cambridge, MA: The MIT Press.

Tanaka, J. S. (1993). Multifaceted Conceptions of Fit in Structural Equation Models. En K. A. Bollen y J. S. Long (Eds.), Testing Structural Equation Models (pp. 10-39). Newbury Park, CA: Sage.

Theurer, K., y Wister, A. (2010). Altruistic Behavior and Social Capital as Predictors of WellBeing among Older Canadians. Aging \& Society, 30, 157-181.

Videra-García, A., y Reigal-Garrido, R. (2013). Autoconcepto físico, percepción de salud y satisfacción vital en una muestra de adolescentes. Anales de Psicología, 29(1), 141-147.

Wang, J., y Wang, X. (2012). Structural Equation Modeling. Applications Using Mplus. West Sussex: Wiley. 